\title{
Photo double ionization of molecular deuterium
}

\author{
T J Reddish $\dagger$ and J M Feagin $\ddagger$ \\ $\dagger$ Physics Department, The University of Newcastle, Newcastle upon Tyne NEI 7RU, UK \\ \$ Department of Physics, California State University-Fullerton, Fullerton, CA 92834, USA \\ Received 4 December 1998
}

\begin{abstract}
A helium-like description of photo double ionization of molecular deuterium is extended to derive a dependence of molecular excitation amplitudes on electron energy sharing and dynamical quantum numbers labelling internal modes of excitation of the escaping electron pair. Both linear and circular polarizations are considered, and predictions regarding circular dichroisms in the angular distributions of the electron and ion pairs are given. A detailed comparison of the model with recent $(\gamma, 2 \mathrm{e})$ measurements is also presented.
\end{abstract}

\section{Introduction}

Photo double ionization (PDI) of molecular deuterium $\mathrm{D}_{2}$-or equivalently molecular hydrogen $\mathrm{H}_{2}$-is challenging because of the inevitable full four-particle fragmentation of the molecule. A thorough study of the resulting few-body escape dynamics clearly requires measurement of differential cross sections, particularly for the lowest photon energies. Recent $(\gamma, 2 \mathrm{e})$ studies of $\mathrm{D}_{2}$ (Reddish et al 1997a, Wightman et al 1998, Scherer et al 1998) have demonstrated a remarkable likeness of $\mathrm{D}_{2}$ and helium triply differential cross sections (TDCS), although the characteristic pair of lobes in the helium distributions are closer together in the molecule and significantly narrower. The two groups' measurements are in excellent agreement and were both obtained for equal electron energy sharing, $E_{1}=E_{2} \sim 10 \mathrm{eV}$, with coplanar detection orthogonal to the photon beam direction. In addition, Dörner et al (1998b) have performed coincidence experiments between the ion pairs and one of the escaping electrons. They thus extracted a one-electron angular asymmetry parameter $\beta_{1}$ as a function of electron energy sharing for parallel and perpendicular ion-axis orientations relative to the photon polarization. They also made the surprising observation that the electron distribution is azimuthally symmetric about the photon polarization axis even when the azimuthal symmetry is broken by a perpendicular ion-axis orientation. These experiments have awakened interest in PDI of these fundamental molecules after a decade of silence following the pioneering ion-ion coincidence experiments on $\mathrm{H}_{2}$ of Dujardin et al (1987) and of Kossmann et al (1989).

We have recently developed a very basic description of the TDCS for diatomic molecules (Feagin 1998) based closely on the PDI amplitude for helium (e.g. Huetz et al 1991, 1995, Lablanquie et al 1995, Maulbetsch and Briggs 1993, Kazansky and Ostrovsky 1995, Pont and Shakeshaft 1995, Feagin 1996) and also on the earlier theoretical studies of Le Rouzo (1986, 1988). We thus derive a helium-like expression for the electron-pair angular distribution which depends on the orientation of the ion axis at the instant of photoionization and therefore on amplitudes for excitation parallel and perpendicular to the molecular axis. When integrated over the momentum of an undetected electron for comparison with the ion-electron results of 
Dörner et al (1998b), we obtain the azimuthally symmetric electron distribution they observed for ion-axis alignments both parallel and perpendicular to the photon polarization axis. When integrated over the alignment of an undetected ion axis for comparison with our $(\gamma, 2 \mathrm{e})$ data and also the data of Scherer et al (1998), we obtain an expression with two contributions: one identical in form to the helium TDCS; and one with an angular distribution independent of the photon polarization direction and proportional to the square of the difference in amplitudes for parallel and perpendicular molecular excitation. The result is in good agreement with measured ratios of the TDCS in $\mathrm{D}_{2}$ to that in He and helps to explain a surprising observed increase in the ratios where the individual angular distributions vanish due to an exact parity-exchange selection rule.

In this paper, we extend our earlier derivation of the molecular photofragmentation amplitude (Feagin 1998) to include a fourth-order Wannier description of the electron-pair continuum wavefunction and thereby obtain an approximate $E_{1}, E_{2}$ energy dependence of the amplitudes for excitation parallel and perpendicular to the molecular axis. At the same time, we attempt to exhibit the role of dynamical excitations internal to the electron pair. Berakdar and Klar (1992) predicted, and Viefhaus et al (1996) and Mergel et al (1998) demonstrated, that PDI in helium with circularly polarized photons leads to a dichroism in the electron-pair angular distribution, and that phase-sensitive probes of the internal electron-pair continuum dynamics are possible (Berakdar et al 1993, see also Berakdar 1998, 1999). Thus, we also extend our description of molecular photofragmentation to include dichroism, a topic with a long and rich history in molecular physics, although we focus on the phenomenon as a tool to probe few-body Coulomb dynamics. Finally, we present further technical details on the comparison of our description with measured TDCS ratios in $\mathrm{D}_{2}$ and $\mathrm{He}$ and provide additional experimental evidence for some of the conclusions we have drawn.

Our theoretical description of the outgoing electron pair is characterized by the collective coordinates defined by the separation vector $\boldsymbol{R}=\boldsymbol{r}_{1}-\boldsymbol{r}_{2}$ and the centre-of-mass (CM) position vector $\boldsymbol{r}=\frac{1}{2}\left(\boldsymbol{r}_{1}+\boldsymbol{r}_{2}\right)$ of the ionized electron pair relative to the $\mathrm{CM}$ of the ion pair. These vectors replace the usual position vectors $\boldsymbol{r}_{1}$ and $\boldsymbol{r}_{2}$ of the electrons relative to the ion-pair CM, so that the dipole excitation operator of the electron pair reduces to simply $\epsilon \cdot r_{1}+\epsilon \cdot r_{2}=2 \epsilon \cdot r$. We thus consider a photon absorbed by the electron-pair $\mathrm{CM}$ and describe the fragmentation state of the system by a Wannier momentum function of the detector coordinates

$$
\begin{aligned}
\boldsymbol{k}_{r} & =\boldsymbol{k}_{1}+\boldsymbol{k}_{2} \quad \boldsymbol{k}_{R}=\frac{1}{2}\left(\boldsymbol{k}_{1}-\boldsymbol{k}_{2}\right) \\
k_{r} & =2 \sqrt{\frac{1}{2} E+\sqrt{E_{1} E_{2}} \cos \theta_{12}} \quad k_{R}=\sqrt{\frac{1}{2} E-\sqrt{E_{1} E_{2}} \cos \theta_{12}}
\end{aligned}
$$

conjugate to $\boldsymbol{r}$ and $\boldsymbol{R}$. Here $\boldsymbol{k}_{1}$ and $\boldsymbol{k}_{2}$ are the conventional detector coordinates, i.e. the momenta conjugate to $\boldsymbol{r}_{1}$ and $\boldsymbol{r}_{2}$, with mutual angle $\theta_{12}=\cos ^{-1}\left(\hat{\boldsymbol{k}}_{1} \cdot \hat{\boldsymbol{k}}_{2}\right)$ and electron energies $E_{i}=\frac{1}{2} k_{i}^{2}$. Then, $E=E_{1}+E_{2}$ is the system excess energy above threshold, an approximate quantity defined by the 'vertical transition' from the molecular ground state to the ion-exploding continuum state.

We will derive and express our results using vector methods independent of a particular angular representation. Then, to derive the angular dependence of a cross section as a function of $\boldsymbol{k}_{r}, \boldsymbol{k}_{R}$ or $\boldsymbol{k}_{1}, \boldsymbol{k}_{2}$, we will only need the polar-angle representation of the momentum vectors relative to lab-fixed axes,

$$
\hat{\boldsymbol{k}}_{i}=\sin \theta_{i} \cos \phi_{i} \hat{\boldsymbol{x}}+\sin \theta_{i} \sin \phi_{i} \hat{\boldsymbol{y}}+\cos \theta_{i} \hat{\boldsymbol{z}}
$$

where $i$ stands for any of the labels $R, r, 1$ or 2 .

We use atomic units throughout, except that we label energies in $\mathrm{eV}$. 


\section{Photoionization cross section}

We retain the axial-recoil description of Feagin (1998) to write down an approximate transition dipole moment describing the excitation and escape of the electron pair from the molecule. We thus assume the ion-axis orientation $\boldsymbol{R}_{N}$ is fixed during the quick escape of the electrons from the instant of photon absorption to the resulting Coulomb explosion of the ion pair (cf Zare 1988). We will generalize this description, however, to arbitrary electron energy sharing $E_{1}$ and $E_{2}=E-E_{1}$ and to include circular dichroism. We thus consider just the Wannier component $\psi_{\boldsymbol{k}_{r} \boldsymbol{k}_{R}}^{W}$ of the electron-pair continuum state $\psi_{\boldsymbol{k}_{r} \boldsymbol{k}_{R}}$ which has been photoexcited from an electron-pair initial state $\psi_{i}$, and define the components of the molecular-frame transition dipole moment according to (primes denote molecular-frame quantities)

$$
\mu_{\alpha}^{\prime} \equiv\left\langle\psi_{\boldsymbol{k}_{r}^{\prime} k_{R}^{\prime}}\left|x_{\alpha}^{\prime}\right| \psi_{i}\right\rangle \simeq \sum_{K}\left\langle\psi_{\boldsymbol{k}_{r}^{\prime} \boldsymbol{k}_{R}^{\prime}} \mid \psi_{L K}^{W}\right\rangle\left\langle\psi_{L K}^{W}\left|x_{\alpha}^{\prime}\right| \psi_{i}\right\rangle,
$$

where $x_{\alpha}^{\prime}=\boldsymbol{r}^{\prime} \cdot \boldsymbol{\epsilon}_{\alpha}^{\prime}$ is a component of the electron-pair CM vector along one of the molecularframe axes $\alpha=x^{\prime}, y^{\prime}, z^{\prime}$ with the $z^{\prime}$ axis along the internuclear axis $\boldsymbol{R}_{N}$. Here $L$ is the total angular momentum quantum number of the electron pair, while $K$ is its projection quantum number along the interelectronic momentum direction $\hat{\boldsymbol{k}}_{R},\left|\boldsymbol{L} \cdot \hat{\boldsymbol{k}}_{R}\right| \equiv K$. Once we have rotated the dipole moment from the molecular frame to the laboratory frame $\boldsymbol{\mu}^{\prime} \rightarrow \boldsymbol{\mu}$, we obtain the molecular PDI amplitude from simply $f\left(\boldsymbol{R}_{N}\right)=\boldsymbol{\epsilon} \cdot \boldsymbol{\mu}$ for a given photon polarization $\boldsymbol{\epsilon}$.

If we take the final state $\left\langle\psi_{\boldsymbol{k}_{r}^{\prime} \boldsymbol{k}_{R}^{\prime}}\right|$ to be simply an outgoing plane-wave detector state, then the overlap $\left\langle\psi_{\boldsymbol{k}_{r}^{\prime} \boldsymbol{k}_{R}^{\prime}} \mid \psi_{L K}^{W}\right\rangle \simeq \tilde{\psi}_{L K}^{W}\left(\boldsymbol{k}_{r}^{\prime}, \boldsymbol{k}_{R}^{\prime}\right)$, i.e. the momentum-space representation (Fourier transform) of the Wannier wavefunction. This momentum wavefunction then characterizes for us the dependence of the cross section on the detector coordinates $\boldsymbol{k}_{r}$ and $\boldsymbol{k}_{R}$. In particular, the internal excitation amplitudes $c_{\alpha K} \equiv\left\langle\psi_{L K}^{W}\left|x_{\alpha}^{\prime}\right| \psi_{i}\right\rangle$ from equation (3) depend only on the excess energy $E$ - fully independent of the momenta $\boldsymbol{k}_{r}$ and $\boldsymbol{k}_{R}$. The rotational symmetry of the diatomic molecule about $\boldsymbol{R}_{N}$ requires that $c_{x^{\prime} K}=c_{y^{\prime} K} \equiv c_{\Pi K}$ and $c_{z^{\prime} K} \equiv c_{\Sigma K}$, where $\Sigma$ and $\Pi$ are the conventional labels distinguishing excitations parallel and perpendicular, respectively, to the molecular axis. In a partial-wave analysis, $\Sigma$ and $\Pi$ also specify perpendicular and parallel projections, respectively, of the electron-pair angular momentum along the molecular axis, $\left|\boldsymbol{L} \cdot \hat{\boldsymbol{R}}_{N}\right|$. In principle, these amplitudes contain all initial state effects and the gauge dependence. Rather than evaluate them here, however, we simply parametrize our results in terms of their ratios.

Following Feagin (1998), we approximate $\tilde{\psi}_{L K}^{W}\left(\boldsymbol{k}_{r}^{\prime}, \boldsymbol{k}_{R}^{\prime}\right)$ by the helium ${ }^{1} \mathrm{P}^{\mathrm{o}}$ momentum distribution so that $K=0,1$ and thereby consider excitation of the dominant ${ }^{1} \mathrm{~S}^{\mathrm{e}}$ component of the molecular ground state $\dagger$. We thus obtain from fourth-order Wannier theory the simple and useful geometrical form (see Feagin 1995, 1996, and also Dörner et al 1998a)

$$
\tilde{\psi}_{L=1, K}^{W}\left(\boldsymbol{k}_{r}^{\prime}, \boldsymbol{k}_{R}^{\prime}\right) \sim \tilde{\Lambda}\left(k_{r}, k_{R}\right) \times \begin{cases}-\boldsymbol{\epsilon}_{\alpha}^{\prime} \cdot \hat{\boldsymbol{k}}_{R}^{\prime} \hat{\boldsymbol{k}}_{R}^{\prime} \cdot \boldsymbol{k}_{r}^{\prime}, & K=0 \\ \boldsymbol{\epsilon}_{\alpha}^{\prime} \cdot \boldsymbol{k}_{r}^{\prime}-\boldsymbol{\epsilon}_{\alpha}^{\prime} \cdot \hat{\boldsymbol{k}}_{R}^{\prime} \hat{\boldsymbol{k}}_{R}^{\prime} \cdot \boldsymbol{k}_{r}^{\prime}, & K=1,\end{cases}
$$

where $\tilde{\Lambda}\left(k_{r}, k_{R}\right)$ is an 'internal' momentum wavefunction. With this approximation the molecular transition dipole moment equation (3) has the simple and useful form

$$
\mu_{\alpha}^{\prime} \simeq \tilde{\Lambda}\left(k_{r}, k_{R}\right)\left(c_{\alpha 1} \boldsymbol{\epsilon}_{\alpha}^{\prime} \cdot \boldsymbol{k}_{r}^{\prime}-\left(c_{\alpha 0}+c_{\alpha 1}\right) \boldsymbol{\epsilon}_{\alpha}^{\prime} \cdot \hat{\boldsymbol{k}}_{R}^{\prime} \hat{\boldsymbol{k}}_{R}^{\prime} \cdot \boldsymbol{k}_{r}^{\prime}\right),
$$

so that if we define a diagonal dyadic $C_{K}^{\prime}$ with diagonal components $c_{x^{\prime} K}=c_{y^{\prime} K}=c_{\Pi K}$ and $c_{z^{\prime} K}=c_{\Sigma K}$, the molecular-frame vector dipole moment can be written conveniently as

$$
\boldsymbol{\mu}^{\prime} \simeq \tilde{\Lambda}\left(k_{r}, k_{R}\right)\left(\boldsymbol{C}_{1}^{\prime} \cdot \boldsymbol{k}_{r}^{\prime}-\left(\boldsymbol{C}_{0}^{\prime}+\boldsymbol{C}_{1}^{\prime}\right) \cdot \hat{\boldsymbol{k}}_{R}^{\prime} \hat{\boldsymbol{k}}_{R}^{\prime} \cdot \boldsymbol{k}_{r}^{\prime}\right)
$$

$\dagger$ Joy and Parr (1958) estimated the ground state components to be $95.8 \% \mathrm{~s}^{2} \mathrm{~S}^{\mathrm{e}}, 2.6 \% \mathrm{sd} \mathrm{D}^{\mathrm{e}}$, and $1.5 \% \mathrm{p}^{2}$. See also Bishop (1963). 
This notation facilitates rotation to the laboratory frame. The transformation is readily accomplished without reference to Euler angles by noting that the molecular-frame diagonal dyadic $\boldsymbol{C}_{K}^{\prime}$ can be expressed as $\boldsymbol{C}_{K}^{\prime}=c_{\Pi K} \mathbf{I}+\left(c_{\Sigma K}-c_{\Pi K}\right) \hat{\boldsymbol{R}}_{N}^{\prime} \hat{\boldsymbol{R}}_{N}^{\prime}$, where $\mathbf{I}$ is the unit dyad. Then, since $\hat{\boldsymbol{R}}_{N}^{\prime} \rightarrow \hat{\boldsymbol{R}}_{N}$ under rotation to the lab frame, $C_{K}^{\prime} \rightarrow \boldsymbol{C}_{K}=c_{\Pi K} \mathbf{I}+$ $\left(c_{\Sigma K}-c_{\Pi K}\right) \hat{\boldsymbol{R}}_{N} \hat{\boldsymbol{R}}_{N}$ under rotation. (Despite the form invariance, $\boldsymbol{C}_{K}$ is nondiagonal.) It follows that the lab-frame dipole moment $\boldsymbol{\mu}$ is form invariant under rotation with all vectors in equation (6) simply replaced by their 'unprimed' lab-frame counterparts. Hence, the molecular photoexcitation amplitude for linear polarization $\epsilon$ is given by

$$
\begin{aligned}
f\left(\boldsymbol{R}_{N}\right)=\boldsymbol{\epsilon} \cdot \boldsymbol{\mu} \simeq \tilde{\Lambda}\left(k_{r}, k_{R}\right)\left[\boldsymbol{\epsilon} \cdot \boldsymbol{C}_{1} \cdot \boldsymbol{k}_{r}-\boldsymbol{\epsilon} \cdot\left(\boldsymbol{C}_{0}+\boldsymbol{C}_{1}\right) \cdot \hat{\boldsymbol{k}}_{R} \hat{\boldsymbol{k}}_{R} \cdot \boldsymbol{k}_{r}\right] \\
=\tilde{\Lambda}\left(k_{r}, k_{R}\right)\left[c_{\Pi 1} \boldsymbol{\epsilon} \cdot \boldsymbol{k}_{r}-\left(c_{\Pi 0}+c_{\Pi 1}\right) \boldsymbol{\epsilon} \cdot \hat{\boldsymbol{k}}_{R} \hat{\boldsymbol{k}}_{R} \cdot \boldsymbol{k}_{r}\right. \\
\left.+\boldsymbol{\epsilon} \cdot \hat{\boldsymbol{R}}_{N}\left(\Delta c_{1} \hat{\boldsymbol{R}}_{N} \cdot \boldsymbol{k}_{r}-\left(\Delta c_{0}+\Delta c_{1}\right) \hat{\boldsymbol{R}}_{N} \cdot \hat{\boldsymbol{k}}_{R} \hat{\boldsymbol{k}}_{R} \cdot \boldsymbol{k}_{r}\right)\right]
\end{aligned}
$$

where $\Delta c_{K} \equiv c_{\Sigma K}-c_{\Pi K}$. We consider circular polarization below. The scalar product $\hat{\boldsymbol{k}}_{R} \cdot \boldsymbol{k}_{r}$ in this result makes clear that detecting $\boldsymbol{k}_{R} \perp \boldsymbol{k}_{r}$ selects pure $K=1$ internal excitation. On the other hand, detecting $\boldsymbol{k}_{R} \| \boldsymbol{k}_{r}$ selects pure $K=0$ internal excitation. In general, the electron pair occupies a $K=0,1$ mixture, although we might expect from studies of helium (Dörner et al 1998a) in the $1-20 \mathrm{eV}$ range that the $K=1$ amplitudes dominate by roughly a factor of three. Likewise, detecting $\boldsymbol{R}_{N} \perp \boldsymbol{\epsilon}$ selects pure $\Pi$ molecular excitation, whereas detecting $\boldsymbol{R}_{N} \| \boldsymbol{\epsilon}$ selects pure $\Sigma$ molecular excitation. As expected, equation (7) reduces to the helium PDI amplitude (cf Dörner et al 1998b) if one ignores $\Sigma$, $\Pi$ differences and sets $c_{\Sigma K}=c_{\Pi K}=c_{K}$.

Equation (7) is easily transformed to conventional detector coordinates $\boldsymbol{k}_{1}$ and $\boldsymbol{k}_{2}$ using equation (1). We thus recover not only the photoexcitation amplitude derived in Feagin (1998),

$$
f\left(\boldsymbol{R}_{N}\right)=\boldsymbol{\epsilon} \cdot \boldsymbol{\mu}=\boldsymbol{\epsilon} \cdot \mathbf{g}\left(1,2, \theta_{12}\right) \cdot \hat{\boldsymbol{k}}_{1}+\boldsymbol{\epsilon} \cdot \mathbf{g}\left(2,1, \theta_{12}\right) \cdot \hat{\boldsymbol{k}}_{2},
$$

where the dyadics are defined as $\mathbf{g}=g_{\Pi} \mathbf{I}+\left(g_{\Sigma}-g_{\Pi}\right) \hat{\boldsymbol{R}}_{N} \hat{\boldsymbol{R}}_{N}$, but also derive an approximate dependence of the amplitudes $g_{\Sigma}, g_{\Pi}$ on the electron-pair energy sharing,

$$
g_{\alpha}\left(i, j, \theta_{12}\right) \simeq \tilde{\Lambda}\left(E_{1}, E_{2}, \theta_{12}\right) \sqrt{2 E_{i}}\left[c_{\alpha 1}-\frac{E_{i}-E_{j}}{2 k_{R}^{2}}\left(c_{\alpha 0}+c_{\alpha 1}\right)\right] .
$$

(Note, $k_{R}^{2}=\frac{1}{2} E-\sqrt{E_{1} E_{2}} \cos \theta_{12}$ from equation (1).) As discussed above, detecting either $\boldsymbol{R}_{N} \| \boldsymbol{\epsilon}$ or $\boldsymbol{R}_{N} \perp \boldsymbol{\epsilon}$ reduces $f\left(\boldsymbol{R}_{N}\right)$ to a helium-like amplitude, either $f \rightarrow g_{\Sigma}\left(1,2, \theta_{12}\right) \boldsymbol{\epsilon} \cdot \hat{\boldsymbol{k}}_{1}+$ $g_{\Sigma}\left(2,1, \theta_{12}\right) \boldsymbol{\epsilon} \cdot \hat{\boldsymbol{k}}_{2}$ or $f \rightarrow g_{\Pi}\left(1,2, \theta_{12}\right) \boldsymbol{\epsilon} \cdot \hat{\boldsymbol{k}}_{1}+g_{\Pi}\left(2,1, \theta_{12}\right) \boldsymbol{\epsilon} \cdot \hat{\boldsymbol{k}}_{2}$. Both of these limits display azimuthal symmetry about the polarization axis $\boldsymbol{\epsilon}$. Although the $\boldsymbol{R}_{N} \| \boldsymbol{\epsilon}$ limit is expected when the azimuthally symmetric initial state is aligned along $\boldsymbol{\epsilon}$, the $\boldsymbol{R}_{N} \perp \boldsymbol{\epsilon}$ limit is at first surprising since the azimuthal symmetry is broken by the initial-state alignment. This result is of course just an artifact of our description which considers excitation of only the dominant ${ }^{1} \mathrm{~S}^{\mathrm{e}}$ groundstate component. Nevertheless, the recent measurements of Dörner et al (1998b) support our $\boldsymbol{R}_{N} \perp \boldsymbol{\epsilon}$ limit.

Equations (7)-(9) give the correct kinematic dependence on the electron-pair vector momenta for a helium-like ${ }^{1} \mathrm{~S}^{\mathrm{e}} \rightarrow{ }^{1} \mathrm{P}^{\mathrm{o}}$ transition, although the internal wavefunction $\tilde{\Lambda}\left(k_{r}, k_{R}\right) \equiv \tilde{\Lambda}\left(E_{1}, E_{2}, \theta_{12}\right)$ has not been derived for molecular fragmentation. The dynamical Wannier saddle in the presence of the molecular axis is too complicated to warrant using the fourth-order internal wavefunction derived for helium (Feagin 1995, 1996, see also Dörner et al 1998a). We therefore simply replace the internal wavefunction with the conventional Gaussian 'correlation function' predicted by second-order Wannier theory in helium. Namely, 
we use

$$
\left|\tilde{\Lambda}\left(E_{1}, E_{2}, \theta_{12}\right)\right|^{2} \equiv G\left(E, \theta_{12}\right) \sim \exp \left(-4 \ln 2 \frac{\left(\pi-\theta_{12}\right)^{2}}{\theta_{1 / 2}^{2}(E)}\right)
$$

with half-width $\theta_{1 / 2}(E)$. In the case of equal-energy sharing, this result has proven extremely useful in parametrizing a variety of helium data even tens of $\mathrm{eV}$ above threshold. The Gaussian wavefunction is a consequence of the harmonic bending motion of the electron pair relative to the ion charge centre, a feature shared by both the atomic and the molecular potentials in lowest order. Although we will not do so here, one might introduce separate correlation functions $G_{\Sigma}$ and $G_{\Pi}$ with differing half-widths for parallel and perpendicular excitations (cf Dörner et al 1998b).

The square of the excitation amplitude $\left|f\left(\boldsymbol{R}_{N}\right)\right|^{2}$ determines the four-body fragmentation distributions. For example, if we integrate over all ion-axis alignments $\hat{\boldsymbol{R}}_{N}$, we derive a TDCS for just the electron pair relevant to the experiments discussed in section 4 . (The cross section is in fact quadruply differential because the Coulomb exploding ions have a small 'FranckCondon' spread in energy of 2-3 eV.) The integration over $\hat{\boldsymbol{R}}_{N}$ is straightforward with the two identities $\int \hat{\boldsymbol{R}}_{N} \hat{\boldsymbol{R}}_{N} \mathrm{~d} \hat{\boldsymbol{R}}_{N}=\frac{4 \pi}{3} \mathbf{I}$ and $\int\left(\boldsymbol{\epsilon} \cdot \hat{\boldsymbol{R}}_{N}\right)^{2} \hat{\boldsymbol{R}}_{N} \hat{\boldsymbol{R}}_{N} \mathrm{~d} \hat{\boldsymbol{R}}_{N}=\frac{4 \pi}{15}(\mathbf{I}+2 \boldsymbol{\epsilon} \boldsymbol{\epsilon})$. We thus generalize Feagin (1998) again to arbitrary electron-pair energy sharing according to

$$
\begin{aligned}
\operatorname{TDCS}\left[\mathrm{D}_{2}\right] \sim & \int|\boldsymbol{\epsilon} \cdot \boldsymbol{\mu}|^{2} \frac{\mathrm{d} \hat{\boldsymbol{R}}_{N}}{4 \pi} \\
= & \frac{2}{15}\left|g_{\Sigma}\left(1,2, \theta_{12}\right) \boldsymbol{\epsilon} \cdot \hat{\boldsymbol{k}}_{1}+g_{\Sigma}\left(2,1, \theta_{12}\right) \boldsymbol{\epsilon} \cdot \hat{\boldsymbol{k}}_{2}\right|^{2} \\
& +\frac{7}{15}\left|g_{\Pi}\left(1,2, \theta_{12}\right) \boldsymbol{\epsilon} \cdot \hat{\boldsymbol{k}}_{1}+g_{\Pi}\left(2,1, \theta_{12}\right) \boldsymbol{\epsilon} \cdot \hat{\boldsymbol{k}}_{2}\right|^{2} \\
& +\frac{6}{15} \operatorname{Re}\left(\left[g_{\Sigma}^{*}\left(1,2, \theta_{12}\right) \boldsymbol{\epsilon} \cdot \hat{\boldsymbol{k}}_{1}+g_{\Sigma}^{*}\left(2,1, \theta_{12}\right) \boldsymbol{\epsilon} \cdot \hat{\boldsymbol{k}}_{2}\right]\right. \\
& \left.\times\left[g_{\Pi}\left(1,2, \theta_{12}\right) \boldsymbol{\epsilon} \cdot \hat{\boldsymbol{k}}_{1}+g_{\Pi}\left(2,1, \theta_{12}\right) \boldsymbol{\epsilon} \cdot \hat{\boldsymbol{k}}_{2}\right]\right) \\
& +\frac{1}{15}\left|\Delta g\left(1,2, \theta_{12}\right) \hat{\boldsymbol{k}}_{1}+\Delta g\left(2,1, \theta_{12}\right) \hat{\boldsymbol{k}}_{2}\right|^{2},
\end{aligned}
$$

where $\Delta g \equiv g_{\Sigma}-g_{\Pi}$. In the limit $g_{\Sigma} \rightarrow g_{\Pi}$, this result reduces as expected to the helium cross section TDCS $[\mathrm{He}]=\left|g\left(1,2, \theta_{12}\right) \boldsymbol{\epsilon} \cdot \hat{\boldsymbol{k}}_{1}+g\left(2,1, \theta_{12}\right) \boldsymbol{\epsilon} \cdot \hat{\boldsymbol{k}}_{2}\right|^{2}$.

We derive doubly differential cross sections (DDCS) for a single electron by integrating the square of the excitation amplitude $\left|f\left(\boldsymbol{R}_{N}\right)\right|^{2}$ from equation (8) or the TDCS from equation (11) over $e i t h e r \hat{\boldsymbol{k}}_{1}$ or $\hat{\boldsymbol{k}}_{2}$. For example, starting with equation (11) and integrating over $\hat{\boldsymbol{k}}_{2}$, we derive the familiar form

$$
\frac{\mathrm{d}^{2} \sigma}{\mathrm{d} E_{1} \mathrm{~d} \hat{\boldsymbol{k}}_{1}}=\frac{1}{4 \pi} \frac{\mathrm{d} \sigma}{\mathrm{d} E_{1}}\left(1+\beta_{1} P_{2}\left(\boldsymbol{\epsilon} \cdot \hat{\boldsymbol{k}}_{1}\right)\right),
$$

where $P_{2}\left(\boldsymbol{\epsilon} \cdot \hat{\boldsymbol{k}}_{1}\right)$ is a second-order Legendre polynomial and $\beta_{1}$ the angular asymmetry parameter. On the other hand, the $\boldsymbol{R}_{N}$-dependent DDCS derived from the square of the excitation amplitude $\left|f\left(\boldsymbol{R}_{N}\right)\right|^{2}$ from equation (8) can be generally expressed in terms of $P_{2}\left(\boldsymbol{\epsilon} \cdot \hat{\boldsymbol{k}}_{1}\right)$ for only the two special geometries $\boldsymbol{R}_{N} \| \boldsymbol{\epsilon}$ or $\boldsymbol{R}_{N} \perp \boldsymbol{\epsilon}$. The former limit is a general result, while the latter is a consequence of our model. In any case, detailed evaluation of $\beta_{1}$ requires a numerical integration because of the complicated angular dependence of the amplitudes $g_{\alpha}\left(i, j, \theta_{12}\right)$ (see Dörner et al 1998b).

We derive an ion angular distribution by integrating the square of the excitation amplitude $\left|f\left(\boldsymbol{R}_{N}\right)\right|^{2}$ from equation (8) over both $\hat{\boldsymbol{k}}_{1}$ and $\hat{\boldsymbol{k}}_{2}$, although it is more straightforward to start with $\left|f\left(\boldsymbol{R}_{N}\right)\right|^{2}$ from equation (7) and integrate over $\hat{\boldsymbol{k}}_{r}$ and $\hat{\boldsymbol{k}}_{R}$. Again, detailed evaluation requires numerical integration. We can derive, however, a useful approximate expression for 
the ion angular asymmetry parameter $\beta_{N}$ by ignoring the weaker angular dependence of the correlation function $G\left(E, \theta_{12}\right)$. The integrals are then easily performed with one of the dyadic identities introduced above equation (11), $\int \hat{\boldsymbol{k}}_{i} \hat{\boldsymbol{k}}_{i} \mathrm{~d} \hat{\boldsymbol{k}}_{i}=\frac{4 \pi}{3} \mathbf{I}$, and with some rearrangement we obtain the familiar form (Dehmer and Dill 1978),

$$
\frac{\mathrm{d} \sigma}{\mathrm{d} \hat{\boldsymbol{R}}_{N}} \propto\left|\boldsymbol{\epsilon} \cdot \boldsymbol{C}_{0}\right|^{2}+2\left|\boldsymbol{\epsilon} \cdot \boldsymbol{C}_{1}\right|^{2} \sim 1+\beta_{N} P_{2}\left(\boldsymbol{\epsilon} \cdot \hat{\boldsymbol{R}}_{N}\right)
$$

where $\beta_{N}=2\left(D_{\Sigma}^{2}-D_{\Pi}^{2}\right) /\left(D_{\Sigma}^{2}+2 D_{\Pi}^{2}\right)$ but now $D_{\alpha}^{2} \equiv\left|c_{\alpha 0}\right|^{2}+2\left|c_{\alpha 1}\right|^{2}$. We compare this quantity with experiment in section 4.

\section{Circular dichroism}

If the absorbed photon is circularly polarized then changing the polarization from left to right $\epsilon_{+} \rightarrow \epsilon_{-}$is tantamount to reflecting the system in a vertical plane passing through the photon propagation direction $\hat{\boldsymbol{k}}_{\gamma}$, which we take to be the laboratory $\hat{\boldsymbol{z}}$ axis with $\boldsymbol{\epsilon}_{ \pm}=\frac{1}{\sqrt{2}}(\hat{\boldsymbol{x}} \pm \mathrm{i} \hat{\boldsymbol{y}})$. Consider the unitary reflection operator defined by

$$
\sigma_{v}|\hat{\boldsymbol{x}}, \hat{\boldsymbol{y}}, \hat{\boldsymbol{z}}\rangle=|\hat{\boldsymbol{x}},-\hat{\boldsymbol{y}}, \hat{\boldsymbol{z}}\rangle
$$

under which the dipole excitation operator transforms according to ( $\sigma_{v}^{\dagger}$ denotes adjoint)

$$
\boldsymbol{\epsilon}_{+} \cdot \boldsymbol{r}=\sigma_{v} \boldsymbol{\epsilon}_{-} \cdot \boldsymbol{r} \sigma_{v}^{\dagger} .
$$

If we reflect for simplicity in the vertical plane defined by $\boldsymbol{k}_{\gamma}$ and the molecular axis $\boldsymbol{R}_{N}$, then the electron-pair initial state is unchanged under reflection, $\sigma_{v}\left|\psi_{i}\left(\hat{\boldsymbol{R}}_{N}\right)\right\rangle=\left|\psi_{i}\left(\hat{\boldsymbol{R}}_{N}\right)\right\rangle$, and the dipole excitation amplitude transforms as

$$
\begin{aligned}
f_{M}\left(\boldsymbol{k}_{r}, \boldsymbol{k}_{R}, \hat{\boldsymbol{R}}_{N}\right) & =\left\langle\psi_{\boldsymbol{k}_{r}, \boldsymbol{k}_{R}}\left(\hat{\boldsymbol{R}}_{N}\right)\left|\boldsymbol{\epsilon}_{M} \cdot \boldsymbol{r}\right| \psi_{i}\left(\hat{\boldsymbol{R}}_{N}\right)\right\rangle \\
& =\left\langle\psi_{\boldsymbol{k}_{r}^{\prime}, \boldsymbol{k}_{R}^{\prime}}\left(\hat{\boldsymbol{R}}_{N}\right)\left|\boldsymbol{\epsilon}_{-M} \cdot \boldsymbol{r}\right| \psi_{i}\left(\hat{\boldsymbol{R}}_{N}\right)\right\rangle=f_{-M}\left(\boldsymbol{k}_{r}^{\prime}, \boldsymbol{k}_{R}^{\prime}, \hat{\boldsymbol{R}}_{N}\right) .
\end{aligned}
$$

Here $\boldsymbol{k}_{r}^{\prime}$ and $\boldsymbol{k}_{R}^{\prime}$ are the momentum vectors expressed along the reflected coordinate axes and therefore with components $\left\{k_{x}^{\prime}, k_{y}^{\prime}, k_{z}^{\prime}\right\}=\left\{k_{x},-k_{y}, k_{z}\right\}$. Thus, changing the circular polarization reflects the cross section in the $\hat{\boldsymbol{x}}, \hat{z}$ plane, and equation (16) relates momentumspace points on opposite sides of the plane. Of course, this result is not due to any intrinsic chirality of molecular deuterium. Rather, the chirality of the photon is transferred to the electron and ion pairs.

It is also clear that one requires at least three distinct directions to establish a handedness, say $\boldsymbol{k}_{\gamma}$ and two of the three vectors $\boldsymbol{k}_{R}, \boldsymbol{k}_{r}, \boldsymbol{R}_{N}$, or $\boldsymbol{k}_{1}, \boldsymbol{k}_{2}, \boldsymbol{R}_{N}$. Otherwise, the reflection is degenerate with a rotational symmetry. For example, if one detects just $\boldsymbol{k}_{1}$ and $\boldsymbol{k}_{2}$, but $k_{1}=k_{2}$, or if all three vectors $\boldsymbol{k}_{1}, \boldsymbol{k}_{2}$, and $\boldsymbol{k}_{\gamma}$ lie in the same plane, then any reflection $\sigma_{v}$ can also be generated by a rotation about $\boldsymbol{k}_{\gamma}$. Likewise, states with $\boldsymbol{k}_{R}$ perpendicular to $\boldsymbol{k}_{r}$ are also reflection-rotation degenerate. This case corresponds again to $k_{1}=k_{2}$, since $\boldsymbol{k}_{r} \cdot \boldsymbol{k}_{R}=E_{1}-E_{2}$ from equation (1). These degeneracies apply to helium as well as to molecular deuterium. However, note that in helium one must detect both electrons to observe dichroism, whereas in the molecule one could detect instead one of the ions and just one of the electrons. In addition, ion-exchange symmetry in $\mathrm{D}_{2}$ simply means that the cross sections are symmetric with respect to $\boldsymbol{R}_{N} \rightarrow-\boldsymbol{R}_{N}$, analogous to the symmetry of singlet electron-pair states under electron exchange $\boldsymbol{k}_{R} \rightarrow-\boldsymbol{k}_{R}$ in both the atom and the molecule. For example, photofragmentation of molecular HD would break this symmetry, though the effect would be very weak.

The dichroism arises from the fact that $\left|f_{+1}\left(\boldsymbol{k}_{r}, \boldsymbol{k}_{R}, \boldsymbol{R}_{N}\right)\right|^{2}-\left|f_{-1}\left(\boldsymbol{k}_{r}, \boldsymbol{k}_{R}, \boldsymbol{R}_{N}\right)\right|^{2}$ is generally nonvanishing, despite the reflection symmetry equation (16). (Note here one 
compares momentum points on the same side of the reflection plane.) Physically, the dichroism means that the photon absorption rate and the resulting electron-ion or electron-pair detection rates will differ if the ion and electron detectors are held fixed and the circular polarization changed. We now relate this fact to coherent excitation of distinct dynamical symmetries.

With the excitation amplitude written as $f_{M}=\epsilon_{M} \cdot \boldsymbol{\mu}$, the dichroism is defined by

$$
\begin{aligned}
\Delta\left(\boldsymbol{R}_{N}\right) & \equiv\left|f_{+1}\left(\boldsymbol{k}_{r}, \boldsymbol{k}_{R}, \boldsymbol{R}_{N}\right)\right|^{2}-\left|f_{-1}\left(\boldsymbol{k}_{r}, \boldsymbol{k}_{R}, \boldsymbol{R}_{N}\right)\right|^{2} \\
& =\boldsymbol{\mu}^{*} \cdot\left(\boldsymbol{\epsilon}_{+}^{*} \boldsymbol{\epsilon}_{+}-\boldsymbol{\epsilon}_{-}^{*} \boldsymbol{\epsilon}_{-}\right) \cdot \boldsymbol{\mu} .
\end{aligned}
$$

This expression is conveniently evaluated in terms of the dyadic

$$
\epsilon_{M}^{*} \epsilon_{M}=\frac{1}{2}\left[\hat{\boldsymbol{x}} \hat{\boldsymbol{x}}+\hat{\boldsymbol{y}} \hat{\boldsymbol{y}}+\mathrm{i}^{M}(\hat{\boldsymbol{x}} \hat{\boldsymbol{y}}-\hat{\boldsymbol{y}} \hat{\boldsymbol{x}})\right] .
$$

The cross term antisymmetric under reflection $\hat{\boldsymbol{y}} \rightarrow-\hat{\boldsymbol{y}}$ delivers the dichroism, while the first term is reflection symmetric. Thus,

$$
\Delta\left(\boldsymbol{R}_{N}\right)=\mathrm{i} \boldsymbol{\mu}^{*} \cdot(\hat{\boldsymbol{x}} \hat{\boldsymbol{y}}-\hat{\boldsymbol{y}} \hat{\boldsymbol{x}}) \cdot \boldsymbol{\mu}=\mathrm{i}\left(\boldsymbol{\mu}^{*} \times \boldsymbol{\mu}\right) \cdot \hat{\boldsymbol{k}}_{\gamma} .
$$

Hence, dichroism is characterized by the pseudovector i $\boldsymbol{\mu}^{*} \times \boldsymbol{\mu}=2 \operatorname{Im} \boldsymbol{\mu} \times \operatorname{Re} \boldsymbol{\mu}$ generated by the transfer of the photon's chirality to the electron and ion pairs.

To give an idea of the momentum dependence of this general expression, we consider two limiting cases of our approximate dipole moment from equation (7). First, we integrate equation (19) over all ion-axis alignments $\hat{\boldsymbol{R}}_{N}$ to derive a helium-like dichroism relevant to experiments which might detect only the electron-pair. The integration is straightforward with just the single identity $\int \hat{\boldsymbol{R}}_{N} \hat{\boldsymbol{R}}_{N} \mathrm{~d} \hat{\boldsymbol{R}}_{N}=\frac{4 \pi}{3} \mathbf{I}$, and we obtain

$$
\begin{aligned}
\int \Delta\left(\boldsymbol{R}_{N}\right) \frac{\mathrm{d} \hat{\boldsymbol{R}}_{N}}{4 \pi} & =2|\tilde{\Lambda}|^{2} \operatorname{Im}\left(\mathcal{C}_{0}^{*} \mathcal{C}_{1}\right) \boldsymbol{k}_{r} \cdot \hat{\boldsymbol{k}}_{R}\left(\hat{\boldsymbol{k}}_{R} \times \hat{\boldsymbol{k}}_{r}\right) \cdot \hat{\boldsymbol{k}}_{\gamma} \\
& =\frac{2}{k_{R}^{2}}|\tilde{\Lambda}|^{2} \operatorname{Im}\left(\mathcal{C}_{0}^{*} \mathcal{C}_{1}\right)\left(E_{1}-E_{2}\right)\left(\boldsymbol{k}_{1} \times \boldsymbol{k}_{2}\right) \cdot \hat{\boldsymbol{k}}_{\gamma} \\
& =\frac{4}{k_{R}^{2}}|\tilde{\Lambda}|^{2} \operatorname{Im}\left(\mathcal{C}_{0}^{*} \mathcal{C}_{1}\right)\left(E_{1}-E_{2}\right) \sqrt{E_{1} E_{2}} \sin \theta_{\gamma 1} \sin \theta_{\gamma 2} \sin \phi_{\gamma 12},
\end{aligned}
$$

where we have used $\boldsymbol{k}_{r} \cdot \boldsymbol{k}_{R}=E_{1}-E_{2}$ and $\boldsymbol{k}_{R} \times \boldsymbol{k}_{r}=\boldsymbol{k}_{1} \times \boldsymbol{k}_{2}$ calculated from equation (1) as well as the polar-angle representation of the momenta $\boldsymbol{k}_{i=1,2}$ from equation (2) with $z$ axis along $\hat{\boldsymbol{k}}_{\gamma}$ such that $\phi_{\gamma 12} \equiv \phi_{\gamma 1}-\phi_{\gamma 2}$. Here $\operatorname{Im}\left(\mathcal{C}_{0}^{*} \mathcal{C}_{1}\right) \equiv \frac{1}{3} \operatorname{Im}\left(c_{\Pi 0}^{*} c_{\Pi 1}+c_{\Pi 0}^{*} c_{\Sigma 1}+c_{\Sigma 0}^{*} c_{\Pi 1}\right)$, which reduces to $\operatorname{Im}\left(c_{0}^{*} c_{1}\right)$ in the helium-like limit $c_{\Sigma K} \rightarrow c_{\Pi K}$. Equation (20) is geometrically identical to the helium dichroism derived by Berakdar and Klar (1992) and studied by Berakdar et al (1993), although it differs in the energy-dependent factors $k_{R}^{-2}\left|\tilde{\Lambda}\left(E_{1}, E_{2}, \theta_{12}\right)\right|^{2}\left(E_{1}-\right.$ $\left.E_{2}\right) \sqrt{E_{1} E_{2}}$, which derive from the fourth-order Wannier theory. The result makes clear that a nonvanishing dichroism in helium requires mixed internal $K$ symmetries corresponding to excitations $\boldsymbol{k}_{r}$ along $\boldsymbol{k}_{R}(K=0)$ and perpendicular to $\boldsymbol{k}_{R}(K=1)$ (see also Berakdar 1998, 1999). In addition, it shows that the dichroism is strongest when $\boldsymbol{k}_{r}$ and $\boldsymbol{k}_{R}$ (or $\boldsymbol{k}_{1}$ and $\boldsymbol{k}_{2}$ ) lie in the same plane with $\boldsymbol{\epsilon}_{M}$, so that $\boldsymbol{k}_{R} \times \boldsymbol{k}_{r}\left(\right.$ or $\left.\boldsymbol{k}_{1} \times \boldsymbol{k}_{2}\right)$ is parallel to $\hat{\boldsymbol{k}}_{\gamma}$. As discussed above, the chirality and hence the dichroism vanish when the fragmentation no longer defines three distinct directions and therefore when any of the three vectors $\boldsymbol{k}_{r}, \boldsymbol{k}_{R}, \hat{\boldsymbol{k}}_{\gamma}\left(\right.$ or $\left.\boldsymbol{k}_{1}, \boldsymbol{k}_{2}, \hat{\boldsymbol{k}}_{\gamma}\right)$ are parallel.

As a final example, we consider equation (19) for equal energy electrons. This case is interesting because the dichroism vanishes in helium when $E_{1}=E_{2}$ (cf equation (20)). The transition dipole moment $\boldsymbol{\mu}$ from equation (7) simplifies considerably when $\boldsymbol{k}_{r} \cdot \boldsymbol{k}_{R}=0$, and one easily obtains

$$
\begin{aligned}
\Delta\left(\boldsymbol{R}_{N}\right)=2|\tilde{\Lambda}|^{2} \operatorname{Im}\left(c_{\Pi 1}^{*} c_{\Sigma 1}\right) \boldsymbol{k}_{r} \cdot \hat{\boldsymbol{R}}_{N}\left(\hat{\boldsymbol{R}}_{N} \times \boldsymbol{k}_{r}\right) \cdot \hat{\boldsymbol{k}}_{\gamma} \\
=2 E G\left(E, \theta_{12}\right) \operatorname{Im}\left(c_{\Pi 1}^{*} c_{\Sigma 1}\right)\left(\hat{\boldsymbol{k}}_{1}+\hat{\boldsymbol{k}}_{2}\right) \cdot \hat{\boldsymbol{R}}_{N}\left[\hat{\boldsymbol{R}}_{N} \times\left(\hat{\boldsymbol{k}}_{1}+\hat{\boldsymbol{k}}_{2}\right)\right] \cdot \hat{\boldsymbol{k}}_{\gamma}
\end{aligned}
$$


Note that here, unlike in helium and in the helium-like result equation (20), only pure $K=1$ internal excitation enters. To emphasize the role of ion detection, we can integrate this result over $\hat{\boldsymbol{k}}_{2}$ to correspond to detection of just one electron. (As mentioned already, a nonvanishing dichroism in helium requires detection of both electrons.) The integration is best performed by taking the $z$ axis along $\boldsymbol{k}_{1}$, so that the correlation function $G\left(E, \theta_{12}\right)$ is azimuthally symmetric. It is then straightforward to show that $\int \Delta\left(\boldsymbol{R}_{N}\right) \mathrm{d} \hat{\boldsymbol{k}}_{2} \propto \hat{\boldsymbol{k}}_{1} \cdot \hat{\boldsymbol{R}}_{N}\left(\hat{\boldsymbol{R}}_{N} \times \hat{\boldsymbol{k}}_{1}\right) \cdot \hat{\boldsymbol{k}}_{\gamma} \rightarrow \frac{1}{2} \sin 2 \phi_{\gamma 1}$ for $\boldsymbol{k}_{1}$ and $\boldsymbol{R}_{N}$ separated by the (azimuthal) angle $\phi_{\gamma 1}$ in a plane perpendicular to $\boldsymbol{k}_{\gamma}$. This is in fact the plane defined by our electron-pair analyser, the description of which we now turn to.

\section{Experiment}

We specialize our molecular electron-pair cross section equation (11) to the case of equal energy sharing $E_{1}=E_{2} \sim 10 \mathrm{eV}$ relevant to our experiments and obtain

$$
\begin{aligned}
\operatorname{TDCS}\left[\mathrm{D}_{2}\right] \sim & \frac{4}{15} \pi\left[2\left|g_{\Sigma}\right|^{2}+7\left|g_{\Pi}\right|^{2}+6 \operatorname{Re}\left(g_{\Pi}^{*} g_{\Sigma}\right)\right]\left(\boldsymbol{\epsilon} \cdot \hat{\boldsymbol{k}}_{1}+\boldsymbol{\epsilon} \cdot \hat{\boldsymbol{k}}_{2}\right)^{2} \\
& +\frac{4}{15} \pi\left|g_{\Sigma}-g_{\Pi}\right|^{2}\left|\hat{\boldsymbol{k}}_{1}+\hat{\boldsymbol{k}}_{2}\right|^{2} .
\end{aligned}
$$

We thus recover the expression derived in Feagin (1998), but now identify $g_{\alpha}\left(E_{1}=E_{2}, \theta_{12}\right) \simeq$ $\sqrt{E G\left(E, \theta_{12}\right)} c_{\alpha 1}$. The first term on the right-hand side of this result is proportional to the equalenergy helium cross section TDCS[He], while the second term is clearly a molecular correction dependent on just the mutual momentum angle, $\left|\hat{\boldsymbol{k}}_{1}+\hat{\boldsymbol{k}}_{2}\right|^{2}=4 \cos ^{2} \frac{1}{2} \theta_{12}$, independent of the polarization vector $\epsilon$. This change in relative azimuthal symmetry about the polarization axis $\epsilon$ is manifest in a relaxing of selection rules which have been established for atomic PDI (Maulbetsch and Briggs 1995). These rules require that the helium TDCS is identically zero in the following situations for a ${ }^{1} \mathrm{P}^{\mathrm{o}}(M=0)$ electron-pair final state: (a) when $k_{1} \perp \epsilon$ and $\boldsymbol{k}_{2} \perp \boldsymbol{\epsilon}$, (b) when $\hat{\boldsymbol{k}}_{1} \cdot \boldsymbol{\epsilon}=-\hat{\boldsymbol{k}}_{2} \cdot \boldsymbol{\epsilon}$, and (c) when $\boldsymbol{k}_{1}=-\boldsymbol{k}_{2} \dagger$.

Rules (a) and (b) are subtle consequences of parity and exchange symmetry of the electron pair and are clearly relaxed in the presence of the additional axis $\boldsymbol{R}_{N}$ leading to the molecular correction term in equation (22) independent of $\epsilon$. Rule (b) results in a null first term on the right-hand side of equation (22) - the helium-like contribution - for $\boldsymbol{k}_{2}$ anywhere on the conical surface shown in figure 1 . Rule (c) on the other hand is a straightforward consequence of parity and exchange symmetry and holds even in the molecule for ungerade, singlet final states. This rule, corresponding to back-to-back electron emission, results in a null molecular cross section along the line $k_{2}=-k_{1}$ on one side of the cone in figure 1. As we have shown (Feagin 1998), the failure of rule (b) in the molecular correction term has measurable consequences in the vicinity of back-to-back emission (i.e. near $\theta_{12}=180^{\circ}$ for coplanar detection geometry) due

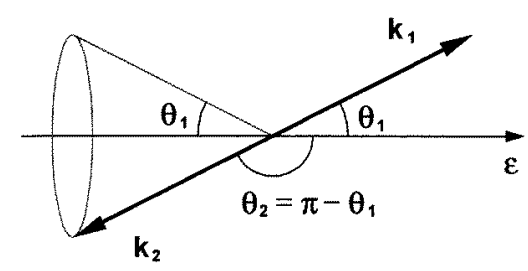

Figure 1. A diagram of the conical surface defined by $\hat{\boldsymbol{k}}_{2} \cdot \boldsymbol{\epsilon}=-\hat{\boldsymbol{k}}_{1} \cdot \boldsymbol{\epsilon}$ on which the helium TDCS vanishes identically from selection rule (b) discussed in the text. This surface, also given by $\theta_{2}=\pi-\theta_{1}$, includes the exact node from parity and exchange considerations for 'back-to-back' electron emission $\theta_{12}=\pi$.

$\dagger$ Note rule (a) holds for all final electron energies, while rules (b) and (c) hold for equal-energy electrons only. 
to the finite detection solid angles resulting in electron collection across a finite portion of the cone defined by rule (b). As we will show here, the lack of a node in the molecular correction term on the opposite side of the cone from $\boldsymbol{k}_{2}=-\boldsymbol{k}_{1}$ (top of the cone in figure 1) also results in a significant increase in the measured molecular TDCS in this angular region in comparison to helium.

For reasons of brevity, the procedures involved in applying the theory to an experimental geometry were only mentioned in Feagin (1998). We therefore present the important details here, not only to make our analysis clear, but also to assist in the theory's application to future measurements. We first consider integration of the cross section equation (22) over the acceptance solid angles defined by our detector. The projections $\boldsymbol{\epsilon} \cdot \hat{\boldsymbol{k}}_{i}$ in equation (22) are generally expressed as $\cos \theta_{i}$ in the conventional spherical polar co-ordinate system with $z$ axis along the polarization axis. Our toroidal analysers, however, are axially symmetric about the photon propagation direction $\boldsymbol{k}_{\gamma}$ and define a detection 'plane' perpendicular to it (see Reddish et al $1997 \mathrm{~b}$ for full details). Therefore integrals over the acceptance solid angles of the detector are more readily performed in a spherical polar co-ordinate system with $z$ axis along $\boldsymbol{k}_{\gamma}$. Then, electron pairs passing into the detector have polar angles $\theta_{\gamma 1} \simeq \theta_{\gamma 2} \simeq 90^{\circ}$ within the range $\pm \frac{1}{2} \delta \theta_{\gamma}$ defined by the analyser's entrance geometry, which is assumed to be constant for all azimuthal angles $\phi_{\gamma_{1}}$ and $\phi_{\gamma^{2}}\left(0^{\circ}\right.$ to $\left.360^{\circ}\right)$ about $\boldsymbol{k}_{\gamma}$. The geometrical upper limit for $\delta \theta_{\gamma}$ is $20^{\circ}\left( \pm \frac{1}{2} \delta \theta_{\gamma}= \pm 10^{\circ}\right)$, but electron optical simulations indicate that a somewhat smaller $\theta_{\gamma}$-angular acceptance is transmitted through the analyser, somewhere between $14^{\circ}$ and $20^{\circ}$ depending on the actual tuning conditions.

We analytically evaluate the $\theta_{\gamma i}$ integrals over the detector slit with a peaking approximation to the Gaussian correlation function $G\left(E, \theta_{12}\right)$. With $\theta_{\gamma 1}=\theta_{\gamma 2}=90^{\circ}$, the projections $\epsilon \cdot \hat{\boldsymbol{k}}_{i}=\cos \phi_{\gamma i}$. We thus set $G\left(E, \theta_{12}\right)=G\left(E,\left|\phi_{\gamma 12} \equiv \phi_{\gamma 1}-\phi_{\gamma_{2}}\right|\right)$ and simply remove it from the integrals. We then obtain for the helium-like contribution $\left(\boldsymbol{\epsilon} \cdot \hat{\boldsymbol{k}}_{1}+\boldsymbol{\epsilon} \cdot \hat{\boldsymbol{k}}_{2}\right)^{2}$ to equation (22)

$$
\begin{aligned}
T_{1}\left(\phi_{\gamma 1}, \phi_{\gamma 2}\right)= & \left(\cos ^{2} \phi_{\gamma 1}+\cos ^{2} \phi_{\gamma 2}\right)\left[\frac{4}{3}\left(2+\cos ^{2} \frac{1}{2} \delta \theta_{\gamma}\right) \sin ^{2} \frac{1}{2} \delta \theta_{\gamma}\right] \\
& +\frac{1}{2} \cos \phi_{\gamma 1} \cos \phi_{\gamma 2}\left(\delta \theta_{\gamma}+\sin \delta \theta_{\gamma}\right)^{2} .
\end{aligned}
$$

As desired, this result reduces to $\delta \theta_{\gamma}^{2}\left(\cos \phi_{\gamma 1}+\cos \phi_{\gamma_{2}}\right)^{2}$ in the small-slit approximation. (Consequently, the $\theta_{\gamma i}$ integrations have little effect on the shape of the angular distributions in helium.) Likewise, we obtain for the $\theta_{\gamma i}$ integrations of the molecular-correction contribution $\left|\hat{\boldsymbol{k}}_{1}+\hat{\boldsymbol{k}}_{2}\right|^{2}=4 \cos ^{2} \frac{1}{2} \theta_{12}$ to equation (22)

$$
T_{2}\left(\phi_{\gamma 1}, \phi_{\gamma 2}\right)=2 \sin ^{2} \frac{1}{2} \delta \theta_{\gamma}+\frac{1}{8} \cos \left(\phi_{\gamma 1}-\phi_{\gamma 2}\right)\left(\delta \theta_{\gamma}+\sin \delta \theta_{\gamma}\right)^{2} .
$$

Although we find these analytic $\theta_{\gamma i}$ integrals $T_{1}$ and $T_{2}$ to be suitably accurate, it was necessary to perform the final $\phi_{\gamma i}$ integrations numerically. Our spectrometer's $\phi_{\gamma}$ angular resolution was quoted in Wightman et al (1998) for the data presented there as $\pm 2.5^{\circ}$ with angular 'bins' of $17^{\circ}$ and $10^{\circ}$ for the two analysers. The $\phi_{\gamma 1}$ and $\phi_{\gamma 2}$ integrals for the data presented here have full-widths of $22^{\circ}$ and $16^{\circ}$, slightly broader than-but in keeping with-the previously quoted values. They were chosen to reproduce the degree of 'filling-in' of the node in the measured helium angular distributions. We denote $T_{1}$ and $T_{2}$ integrated over $\phi_{\gamma i}$ as $\bar{T}_{1}$ and $\bar{T}_{2}$.

Finally, the quantities $\bar{T}_{1}$ and $\bar{T}_{2}$, fully integrated over detection solid angles, can be combined in the usual way to take into account the degree of linear polarization, namely TDCS $\propto \frac{1+S_{1}}{2}$ TDCS $_{x}+\frac{1-S_{1}}{2}$ TDCS $_{y}$ in terms of the Stoke's parameter $S_{1}$ (Schwarzkopf et al 1993).

The ratio of angular distributions $\overline{\mathrm{TDCS}}\left[\mathrm{D}_{2}\right] / \overline{\mathrm{TDCS}}[\mathrm{He}]$ emphasizes their difference, which as figure $2(a)$ demonstrates is most pronounced in the region $\theta_{12}=180^{\circ}$. This peak in the ratio occurs in an angular range where TDCS[D $\left[\mathrm{D}_{2}\right]$ and TDCS$[\mathrm{He}]$ are both very small, as 

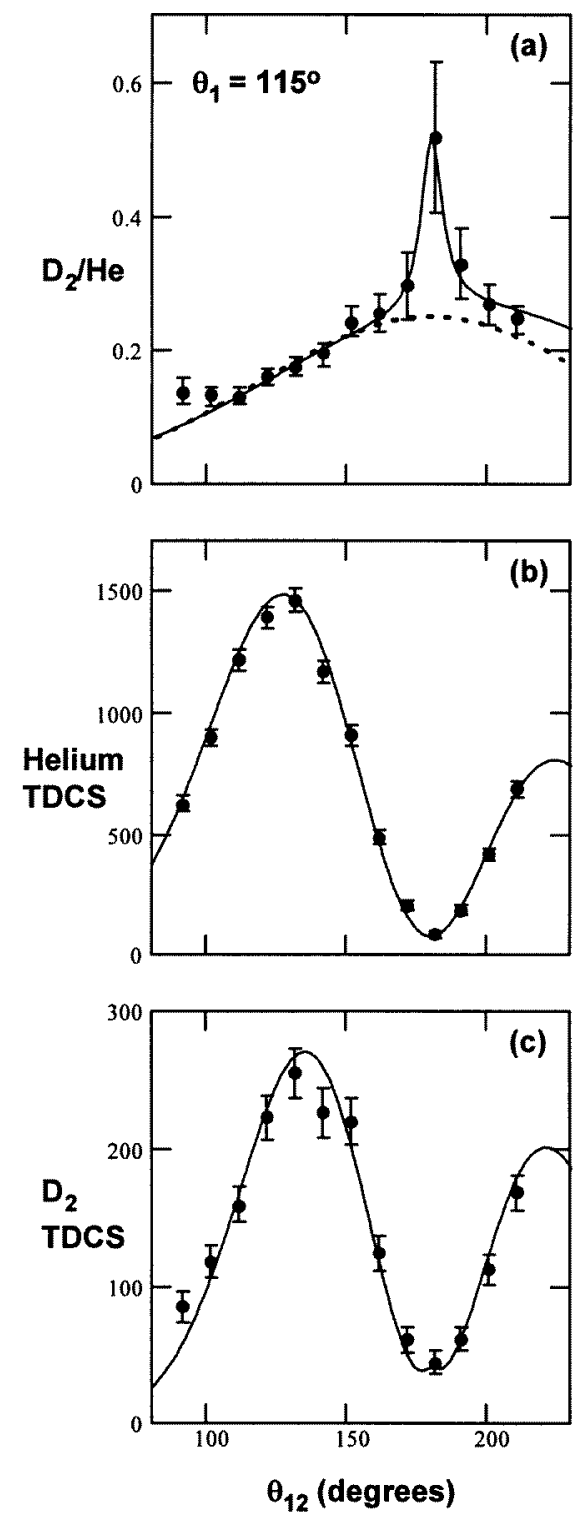

Figure 2. (a) Ratio of the $\mathrm{D}_{2}$-to-He coincidence yield as a function of the electron-pair mutual momentum angle $\theta_{12}$ from the data of Wightman et al (1998) for $\theta_{1}=115^{\circ}$. The solid curve shows the corresponding ratios computed from the theoretical fits from equation (25). The dotted curve under the peak shows the ratio of the two Gaussian correlation functions used for $\mathrm{D}_{2}$ and He. $(b)$ The helium and $(c)$ the $\mathrm{D}_{2}$ TDCS data from Wightman et al (1998) with $E_{1}=E_{2} \simeq 10 \mathrm{eV}$ and Stoke's parameter $S_{1}=0.67 \pm 0.03$. The helium fit includes a Gaussian correlation function with half-width $\theta_{1 / 2}=91^{\circ} \pm 3^{\circ}$. The $\mathrm{D}_{2}$ fit is from equation (22) with a ratio $\eta=g_{\Pi} / g_{\Sigma}=$ -2.1 and correlation function half width $\theta_{1 / 2}=76^{\circ} \pm 3^{\circ}$. Both fits include integration over detection solid angles, as discussed in the text.

figures 2(b) and (c) show, which explains the relatively large error bars. Collecting results, we can express this ratio as

$$
\frac{\overline{\operatorname{TDCS}}\left[\mathrm{D}_{2}\right]}{\overline{\mathrm{TDCS}}[\mathrm{He}]} \sim \frac{G_{\mathrm{D}_{2}}\left(\left|\phi_{\gamma 12}\right|\right)}{G_{\mathrm{He}}\left(\left|\phi_{\gamma 12}\right|\right)}\left[1+C(\eta) \frac{\bar{T}_{2}\left(\phi_{\gamma 1}, \phi_{\gamma 2}\right)}{\bar{T}_{1}\left(\phi_{\gamma 1}, \phi_{\gamma 2}\right)}\right],
$$

where $\phi_{\gamma 12} \equiv \phi_{\gamma 1}-\phi_{\gamma 2}$ and $\eta \equiv g_{\Pi} / g_{\Sigma}=c_{\Pi 1} / c_{\Sigma 1}$ with

$$
C(\eta) \equiv \frac{4|1-\eta|^{2}}{2+7|\eta|^{2}+6 \operatorname{Re} \eta}
$$

Note that when $\eta=1$ corresponding to $g_{\Pi}=g_{\Sigma}, C(1)=0$ and the molecular-correction term vanishes from equation (25), leaving us with simply the ratio of the two Gaussian correlation 
functions. The quantities $\eta$ and $C(\eta)$ are generally complex valued, although we assume both to be real here for simplicity. Using all three data sets $\theta_{1}=98^{\circ}, 115^{\circ}$, and $132^{\circ}$ from Wightman et al (1998) and the $\phi_{\gamma i}$-angular acceptances given above, we obtain a best fit to the ratio data in figure $2(a)$ with $\delta \theta_{\gamma}=18^{\circ} \pm 1^{\circ}$ and $C(\eta)=-1.9 \pm 0.4$, which corresponds to $\eta=-2.1 \pm 0.5$ or $\eta=+0.01 \pm 0.05 \dagger$. These values were used to produce the other curves in figure 2.

We can also relate the ratio $\eta$ to the ion angular asymmetry parameter from equation (13) via the approximation

$$
\beta_{N} \simeq \frac{2\left(1-|\eta|^{2}\right)}{1+|\eta|^{2}} \quad \text { or } \quad|\eta|^{2} \simeq \frac{2-\beta_{N}}{2\left(1+\beta_{N}\right)}
$$

where we have assumed that $\frac{1}{2}\left|c_{\alpha 0} / c_{\alpha 1}\right|^{2} \ll 1$, since analysis of helium data for $E=20 \mathrm{eV}$ has shown that $c_{0} / c_{1} \sim \frac{1}{3}$ (Dörner et al 1998a). Thus, the value $\eta=+0.01 \pm 0.05$ determined in the above fit implies a negligible $g_{\Pi}$ amplitude with $\beta_{N} \simeq+2$, values which are inconsistent with the ion distributions measured by Kossmann et al (1989), who obtained the result $\beta_{N}=-0.71 \pm 0.05$ at a photon energy of $71 \mathrm{eV}$. (Dörner et al $1998 \mathrm{~b}$ obtained the value $\beta_{N}=-0.60 \pm 0.05$ at a photon energy of $58.8 \mathrm{eV}$.) However, the other value $\eta=-2.1 \pm 0.5$ determined in the above fit corresponds to $\beta_{N}=-0.69 \pm 0.13$, which is in good agreement with the Kossmann result. We find this correspondence satisfactory despite the approximations, as a more accurate fit by the above procedure would be inherently difficult because the analysis depends on the ratio of two 'null' signals. This demonstrates that within the assumptions of our approximation, one can use the measured $\beta_{N}$ values to determine the ratio $g_{\Pi} / g_{\Sigma}$ at different photon energies. The ratio peaks around +2.3 for photon energies near the nominal double ionization threshold of $51.1 \mathrm{eV}$ and gradually falls off at higher photon energies to about +1.5 at $100 \mathrm{eV}$. This trend might serve as a rough prediction for future $(\gamma, 2 \mathrm{e})$ measurements and it will be interesting to compare them with future theoretical and experimental studies of both equal and unequal electron energy sharing.

Arguably, the most important evidence in support of our description comes from examining other consequences of the molecular-correction term in the TDCS. Given a ratio of $g_{\Pi} / g_{\Sigma} \simeq$ -2.1 with our kinematic conditions, we can use equation (22) (which includes the assumption that the correlation half-width $\theta_{1 / 2}$ is the same for both $g_{\Pi}$ and $g_{\Sigma}$ amplitudes) to examine the shape of the TDCS at other $\theta_{1}\left(=\phi_{\gamma 1}\right)$ angles. The amplitudes of the two characteristic lobes are a sensitive function of $\theta_{1}$ as well as of the degree of linear polarization $S_{1}$. The effect of the molecular correction term is to dramatically alter the relative intensity of the two lobes as a function of $\theta_{1}$ in comparison to that of helium. This is particularly evident at $\theta_{1}=144^{\circ}$, an angle where the TDCS had been measured earlier (Reddish et al 1997a). The comparison of the TDCS, using both atomic and molecular descriptions but with the same correlation function half-widths $\theta_{1 / 2}=76^{\circ}$, is shown in figures 3 and 4 .

Figure 3(a) shows a polar plot of the Gaussian correlation function $G$ with a $76^{\circ}$ half-width along with the He and $\mathrm{D}_{2}$ angular functions defined by $\overline{\mathrm{TDCS}}[\mathrm{He}] / G$ and $\overline{\mathrm{TDCS}}\left[\mathrm{D}_{2}\right] / G$. The helium distribution is symmetric about the polarization axis while the $\mathrm{D}_{2}$ distribution is clearly skewed by the additional molecular correction term. The product of the correlation function and the two angular functions is shown in figure $3(b)$. The marked increase in amplitude of the smaller lobe along the polarization direction is a consequence of the relaxation of the selection rules we have discussed. As seen in figure 4, this prediction is in good agreement with earlier data, which were taken with $S_{1}=0.67 \pm 0.03$. This supporting evidence for our

$\dagger$ Note $\eta$ as function of $C$ is double valued, and we therefore include the $\eta>0$ value, although our own partial-wave analysis indicates that $\eta<0$ is expected. 


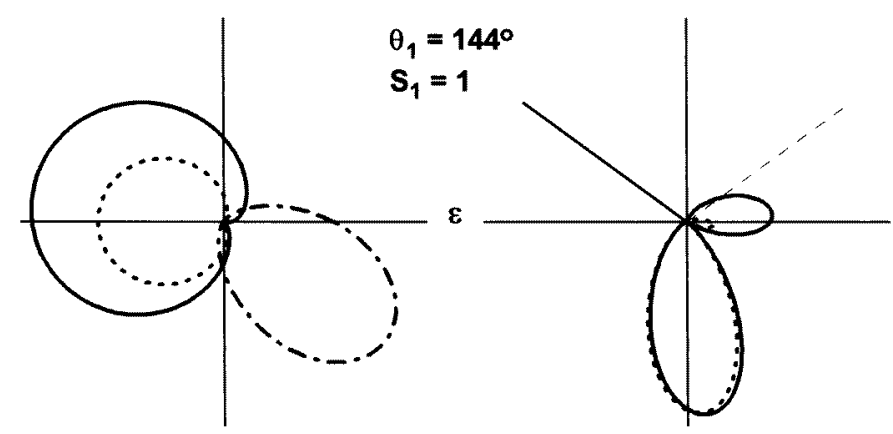

Figure 3. (a) A polar plot for $\theta_{1}=\phi_{\gamma 1}=144^{\circ}$ of the Gaussian correlation function $G\left(E,\left|\phi_{\gamma 1}-\phi_{\gamma 2}\right|\right)$ with half-width $\theta_{1 / 2}=76^{\circ}$ (dash-dot) and the helium (dot) and $\mathrm{D}_{2}$ (solid) angular distributions defined by $\overline{\mathrm{TDCS}}[\mathrm{He}] / G$ and $\overline{\mathrm{TDCS}}\left[\mathrm{D}_{2}\right] / G$. With an amplitude ratio $g_{\Pi} / g_{\Sigma}=-2.1$, the $\mathrm{D}_{2}$ function no longer has symmetry about the polarization axis along the horizontal. (b) A polar plot of the product of the angular distributions and the correlation function with $76^{\circ}$ half-width for $\mathrm{D}_{2}$ (solid) and $\mathrm{He}$ (dots), representing the TDCS with $100 \%$ linear polarization. The solid line indicates the $\theta_{1}$ angle and the dashed line shows the position of the extra node in helium which effectively suppresses the TDCS between $\phi_{\gamma 2}=\pi \pm \phi_{\gamma 1}$.

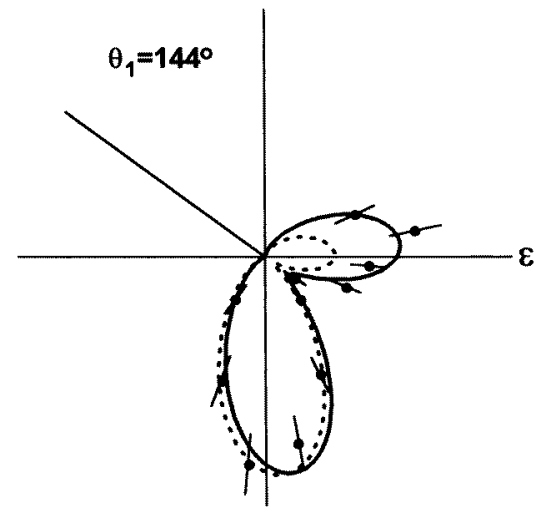

Figure 4. Measured $(\gamma, 2 \mathrm{e}) \mathrm{D}_{2}$ TDCS for $E_{1}=E_{2} \sim 10 \mathrm{eV}$ with Stoke's parameter $S_{1}=0.67 \pm 0.03$ from Reddish et al (1997a). The solid and dotted curves are the molecular and atomic forms of the $\overline{\text { TDCS}}$, respectively, with the same $76^{\circ}$ correlation-function half-width. Both curves include integration over detection solid angles and are normalized at their peak values.

molecular photofragmentation description also suggests that the correlation half-width is not too dissimilar for the $g_{\Pi}$ and $g_{\Sigma}$ amplitudes at these kinematic conditions.

\section{Conclusion}

We have presented a helium-like description of PDI of molecular deuterium-or hydrogenfor both linearly and circularly polarized photons and therefore for circular dichroism in the angular distribution of the ions and electrons. Our simple approach affords an anglefree representation of the cross section that exhibits explicitly its dependence on the electron momenta and the ion-axis orientation, as well as the amplitudes $g_{\Sigma}$ and $g_{\Pi}$ for electron-pair excitation parallel and perpendicular to the molecular axis. By connecting with a fourth-order Wannier description of the electron-pair continuum wavefunction, we derive an approximate dependence of these amplitudes on electron energy sharing and a dynamical quantum number $K$ to classify the internal excitations of the electron pair. The description has thus shown considerable consistency with recent $(\gamma, 2 \mathrm{e})$ and ion-electron experiments.

Our model is based on the dominant $(95.6 \%) \mathrm{S}^{\mathrm{e}}$ component of the molecular ground state 
and neglects entirely higher-order multipoles, although experimental observations to date do not rule them out and recent calculations by Walter and Briggs (1999) show evidence for them with small additional structures in the TDCS. Their 5C calculations of the fragmenting four-particle molecular state are in full analogy with $3 \mathrm{C}$ calculations of helium PDI and are likely to become the 'virtual reality' of molecular PDI. The higher-order multipoles may also account for the observed narrowing of the characteristic lobes in the angular distributions, also seen in the results of Walter and Briggs. While our model's angle-independent simplicity might survive inclusion of the next strongest $(2.6 \%) \mathrm{D}^{\mathrm{e}}$ component, systematic inclusion of higher multipoles would likely require a more traditional approach involving a full partial-wave analysis.

We have assumed that the correlation function half-width $\theta_{1 / 2}$ is the same for both amplitudes $g_{\Sigma}$ and $g_{\Pi}$. Although this approximation seems acceptable when examining the shape of the angular distribution in the vicinity of the node at $\theta_{12}=\pi$, where the correlation functions are peaked, it becomes questionable when considering the overall shape of the TDCS. Extensions of our description might therefore include construction of a Wannier wavefunction for an ion pair with the ground-state separation of the molecule and an electron pair with outgoing components parallel and perpendicular to the ion axis.

\section{Acknowledgments}

The $(\gamma, 2 \mathrm{e})$ experiments of Reddish and co-workers have been supported by the EPSRC(UK). The theoretical work of Feagin and co-workers has been supported by the US Department of Energy, Division of Chemical Science, Fundamental Interactions Branch, Office of Basic Energy Science and Energy Research. This work also benefited from a NATO travel grant for JMF for an extended visit with John Briggs and co-workers at Freiburg University.

\section{References}

Berakdar J 1998 J. Phys. B: At. Mol. Opt. Phys. 313167

1999 J. Phys. B: At. Mol. Opt. Phys. 32 L27

Berakdar J and Klar H 1992 Phys. Rev. Lett. 691175

Berakdar J, Klar H, Huetz A and Selles P 1993 J. Phys. B: At. Mol. Opt. Phys. 261463

Bishop D M 1963 Mol. Phys. 6305

Dehmer J L and Dill D 1978 Phys. Rev. A 18164

Dörner R et al 1998a Phys. Rev. A 571074

-1998b Phys. Rev. Lett. 815776

Dujardin G, Besnard M J, Hellner L and Malinovitch Y 1987 Phys. Rev. A 355012

Feagin J M 1995 J. Phys. B: At. Mol. Opt. Phys. 281495

1996 J. Phys. B: At. Mol. Opt. Phys. 29 L551

-1998 J. Phys. B: At. Mol. Opt. Phys. 31 L729

Huetz A, Andric L, Jean A, Lablanquie P, Selles P and Mazeau J 1995 XIX Int. Conf. on Physics of Electronic and Atomic Collisions, Invited Talk (AIP Conf. Proc. 139)

Huetz A, Selles P, Waymel D and Mazeau J 1991 J. Phys. B: At. Mol. Opt. Phys. 241917

Joy H W and Parr R G 1958 J. Chem. Phys. 28448

Kazansky A K and Ostrovsky V N 1995 Phys. Rev. A 513698

Kossmann H, Schwarzkopf O, Kämmerling B and Schmidt V 1989 Phys. Rev. Lett. 632040

Lablanquie P, Mazeau J, Andric L, Selles P and Huetz A 1995 Phys. Rev. Lett. 742192

LeRouzo H 1986 J. Phys. B: At. Mol. Phys. 19 L677

-1988 Phys. Rev. A 371512

Maulbetsch F and Briggs J S 1993 J. Phys. B: At. Mol. Opt. Phys. 26 L647

-1995 J. Phys. B: At. Mol. Opt. Phys. 28551

Mergel V et al 1998 Phys. Rev. Lett. 805301

Pont M and Shakeshaft R 1995 J. Phys. B: At. Mol. Opt. Phys. 28 L571 
-1995 Phys. Rev. A 51 R2676

Reddish T J, Richmond G, Bagley G W, Wightman J P and Cvejanović S 1997b Rev. Sci. Instrum. 682685

Reddish T J, Wightman J P, MacDonald M A and Cvejanović S 1997a Phys. Rev. Lett. 792438

Scherer N, Lörch H and Schmidt V 1998 J. Phys. B: At. Mol. Opt. Phys. 31 L817

Schwarzkopf O, Krässig B, Elmiger J and Schmidt V 1993 Phys. Rev. Lett. 703008

Viefhaus J et al 1996 Phys. Rev. Lett. 773975

Walter M and Briggs J S 1999 J. Phys. B: At. Mol. Opt. Phys. 322487

Wightman J P, Cvejanović S and Reddish T J 1998 J. Phys. B: At. Mol. Opt. Phys. 311753

Zare R N 1988 Angular Momentum (New York: Wiley-Interscience) 